\title{
FOURIER TRANSFORM TO SOLVE INFINITE STRING FREE VIBRATION OF APPLICATION
}

\author{
Xiaofeng Li ${ }^{*}$, Xiaoxu Dong ${ }^{2}$, Lihua Zhang ${ }^{3}$ \\ ${ }^{1 * X i a o f e n g ~ L i, ~ S c h o o l ~ o f ~ S c i e n c e s, ~ S o u t h w e s t ~ P e t r o l e u m ~ U n i v e r s i t y, ~ C h e n g d u, S i c h u a n ~ 610500, C h i n a ~}$ \\ ${ }^{2}$ Xiaoxu Dong, School of Sciences, Southwest Petroleum University, Chengdu,Sichuan 610500,China \\ ${ }^{3}$ Lihua Zhang, No. 2 Middle School,Dingxi,Gansu 730500,China
}

*Corresponding Author: -

\begin{abstract}
:
This paper presents the definite solution of equations of infinite string free vibration problem and the establishment of conditions, given the definite solution to solve the problem of two algorithms based on Fourier transform for solving partial differential equations are general steps. The problem-solving differential equations after some converted into solving the problems of ordinary differential equations, and then to ask as a function of quadrature or inverse solution to give the original solution to the problem.
\end{abstract}

Keywords: Unbounded string Fourier transform D'Alembert's formula algorithm 


\section{INFINITE STRING FREE VIBRATION PROBLEMS}

Infinite string free vibration problems is the partial differential equation satisfies the following conditions:

$$
\left\{\begin{array}{l}
\frac{\partial^{2} u}{\partial t^{2}}=a^{2} \frac{\partial^{2} u}{\partial x^{2}} \\
u(x, 0)=\varphi(x), \quad(-\infty<x<+\infty, t>0) \\
u_{t}(x, 0)=\psi(x)
\end{array}\right.
$$

For the partial differential equations, we can present major step Fourier transform method of solving:

1) Equations and boundary conditions of the Fourier transform, using the differential nature of Fourier transform to obtain a definite solution of the problem concerning the unknown function as a function of ordinary differential equations;

2) Solving the problem of definite solution of ordinary differential equations, the unknown function like function;

3) As a function of the resulting inverse transform, to obtain the solution of the original solution to the problem.

\section{Fourier transform and properties}

\subsection{Fourier transform concept}

Fourier transform

$$
F(w)=F[f(x)]=\int_{-\infty}^{+\infty} f(x) e^{i w x} d x
$$

Inverse Fourier transform

$$
f(x)=F^{-1}[F(w)]=\frac{1}{2 \pi} \int_{-\infty}^{+\infty} F(w) e^{-i w x} d x
$$

\subsection{Properties of the Fourier transform ${ }^{[1]}$}

Property 1 (linear nature) Fourier transform is a linear transform, i.e., for an arbitrary complex number $\alpha, \beta$, And function $f_{1}, f_{2}$, The following holds

$$
F\left[\alpha f_{1}+\beta f_{2}\right]=\alpha F\left[f_{1}\right]+\beta F\left[f_{2}\right]
$$

Property 2 (convolution property) $f_{1}(x)$ and $f_{2}(x)$ Fourier transform is equal to the convolution $f_{1}(x)$ and $f_{2}(x)$ the Fourier transform of the product, namely

$$
F\left[f_{1} * f_{2}\right]=F\left[f_{1}\right] \cdot F\left[f_{2}\right] .
$$

Property $3 f_{1}(x)$ and $f_{2}(x)$ is equal to the product of the Fourier transform $f_{1}(x)$ and $f_{2}(x)$ of the Fourier transform of the convolution is multiplied by $\frac{1}{2 \pi}$, that is,

$$
F\left[f_{1} \cdot f_{2}\right]=\frac{1}{2 \pi} F\left[f_{1}\right] \cdot F\left[f_{2}\right]
$$

Property 4 (Differential property) If $f(x), f^{\prime}(x)$ can be Fourier transform, when $|x| \rightarrow \infty, f(x) \rightarrow 0$, The establishment of relations

$$
F\left[f^{\prime}(x)\right]=i \lambda F[f(x)] .
$$

Property 5 If $f(x)$ and $x f(x)$ can be Fourier transform, then

$$
F[-i \lambda f(x)]=\frac{d}{d \lambda} F[f] .
$$

Property $6 F\left[f\left(x \pm x_{0}\right)\right]=e^{ \pm i \lambda x_{0}} F[f(x)]$,

It indicates that the function $f(x)$ along the x-axis to the left or right of the Fourier transform of the displacement $x_{0}$, Equal to $f(x)$ Fourier transform multiplied by a factor $e^{i \lambda x_{0}}$ or $e^{-i \lambda x_{0}}$.

\section{Property 7 (Integral Property)}

If $\lim _{x \rightarrow+\infty} \int_{-\infty}^{x} f(x) d x=0$, then

$$
F\left[\int_{-\infty}^{x} f(x) d x\right]=\frac{1}{i \lambda} F[f(x)] .
$$




\section{The Fourier transform of the two algorithms}

\subsection{Algorithm 1 (conventional solver)}

i. Set $u(x, t), \varphi(x), \psi(x)$ respectively, on the Fourier transform variable $x$ obtained $\tilde{u}(\lambda, t), \tilde{\varphi}(\lambda), \tilde{\psi}(\lambda)$

$$
F[u(x, t)]=\tilde{u}(\lambda, t), \quad F[\phi(x)]=\tilde{\phi}(\lambda), \quad F[\psi(x)]=\tilde{\psi}(\lambda) .
$$

ii.The Fourier transform, reach conclusion

$$
F[u(x, t)]=\tilde{u}(\lambda, t)=\int_{-\infty}^{+\infty} u(x, t) e^{-i \lambda x} d x .
$$

iii. the differential properties of the Fourier transform, reach conclusion

$$
\begin{gathered}
F\left(\frac{\partial^{2} u}{\partial x^{2}}\right)=(i \lambda)^{2} F(u(x, t))=-\lambda^{2} \tilde{u}(\lambda, t), \\
F\left(\frac{\partial^{2} u}{\partial t^{2}}\right)=\int_{-\infty}^{+\infty} \frac{\partial^{2} u}{\partial t^{2}} \cdot e^{-i \lambda x} d x=\frac{d^{2}}{d t^{2}} \int_{-\infty}^{+\infty} u(x, t) \cdot e^{-i \lambda x} d x=\frac{d^{2} \tilde{u}}{d t^{2}} .
\end{gathered}
$$

iv.To the equation (1) on both sides of the Fourier transform, reach conclusion

$$
\frac{d^{2} \tilde{u}}{d t^{2}}=a^{2} \cdot\left(-\lambda^{2}\right) \cdot \tilde{u}(\lambda, t)=-a^{2} \lambda^{2} \tilde{u}(\lambda, t)
$$

Thus,

$$
\frac{d^{2} \tilde{u}}{d t^{2}}+a^{2} \lambda^{2} \tilde{u}(\lambda, t)=0
$$

v. (4) is a second-order ordinary differential equation with parameters $\lambda$, Like

$$
y^{\prime \prime}+a^{2} \lambda^{2} y=0
$$

Its characteristic equation $r^{2}+a^{2} \lambda^{2}=0$, the solution is $r= \pm a \lambda i$, thus, the general solution (5) is $y=c_{1} e^{i a \lambda t}+c_{2} e^{-i a \lambda t} \quad\left(c_{1}, c_{2} \in R\right)$.

Namely, the general solution (4) is $\tilde{u}(\lambda, t)=A(\lambda) e^{-i a \lambda t}+B(\lambda) e^{i a \lambda t}$ Insert the initial conditions (2) (3) to (6), the result is

$$
\left\{\begin{array}{l}
A(\lambda)=\frac{1}{2} \tilde{\varphi}(\lambda)+\frac{1}{2 a} \cdot \frac{1}{i \lambda} \tilde{\psi}(\lambda) \\
B(\lambda)=\frac{1}{2} \tilde{\varphi}(\lambda)-\frac{1}{2 a} \cdot \frac{1}{i \lambda} \tilde{\psi}(\lambda)
\end{array}\right.
$$

Insert the (8) to equation (6), the result is

$$
\tilde{u}(\lambda, t)=\frac{1}{2}\left[\tilde{\varphi}(\lambda) e^{i a \lambda t}+\tilde{\varphi}(\lambda) e^{-i a \lambda t}\right]+\frac{1}{2 a}\left[\frac{\tilde{\psi}(\lambda)}{i \lambda} e^{i a \lambda t}-\frac{\tilde{\psi}(\lambda)}{i \lambda} e^{-i a \lambda t}\right]
$$

vi. Inverse transformation of the resulting image function

$$
u(x, t)=F^{-1}[\tilde{u}(\lambda, t)]
$$

By using the displacement properties of Fourier transform, the result is

$$
\begin{gathered}
F^{-1}\left[\tilde{\varphi}(\lambda) e^{i a \lambda t}\right]=\varphi(x+a t), \\
F^{-1}\left[\tilde{\varphi}(\lambda) e^{-i a \lambda t}\right]=\varphi(x-a t) .
\end{gathered}
$$

By using the integral property of Fourier transform, the result is

$$
\begin{aligned}
& F^{-1}\left[\frac{\tilde{\psi}(\lambda)}{i \lambda} e^{i a \lambda t}\right]=\int_{-\infty}^{x+a t} \psi(\zeta) d \zeta, \\
& F^{-1}\left[\frac{\tilde{\psi}(\lambda)}{i \lambda} e^{-i a \lambda t}\right]=\int_{-\infty}^{x-a t} \psi(\zeta) d \zeta
\end{aligned}
$$


In summary,

$$
\begin{aligned}
u(x, t) & =\frac{1}{2}[\varphi(x+a t)+\varphi(x-a t)]+\frac{1}{2 a}\left[\int_{-\infty}^{x+a t} \psi(\zeta) d \zeta-\int_{-\infty}^{x-a t} \psi(\zeta) d \zeta\right] \\
& =\frac{1}{2}[\varphi(x+a t)+\varphi(x-a t)]+\frac{1}{2 a} \int_{x-a t}^{x+a t} \psi(\zeta) d \zeta .
\end{aligned}
$$

This is the solution to the problem of the definite solution of the free vibration of an unbounded string. This formula is the D'Alembert's formula.

\section{2 algorithm 2 (using matlab program) ${ }^{[2]}$}

Steps i-iv are same with algorithm 1. v. with MATLAB solution.

Input in the MATLAB window (with U instead of $u$, with R1instead of $\tilde{\varphi}(\lambda)$, , with R2 instead of $\tilde{\psi}(\lambda)$ ),

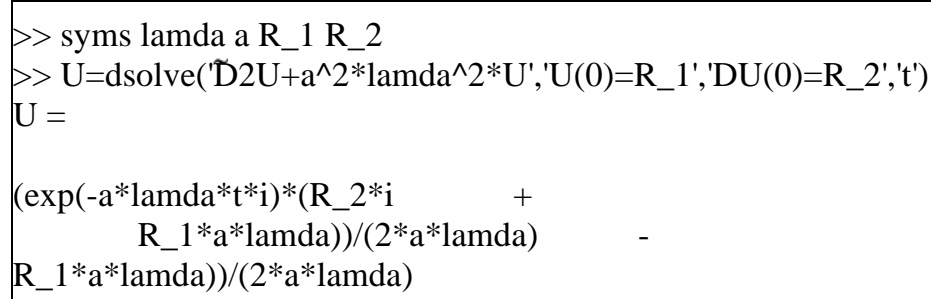

Using Euler's formula $e^{ \pm i x}=\cos x \pm i \sin x$ Sort on, the result is

$$
\tilde{u}(\lambda, t)=\frac{\sin \lambda a t}{\lambda a} \tilde{\psi}(\lambda)+\cos \lambda a t \tilde{\varphi}(\lambda) .
$$

vi. Inverse transformation of the resulting image function $u(x, t)=F^{-1}[\tilde{u}(\lambda, t)]$,

Make the function $\tilde{u}(\lambda, t)$ on the $\lambda$ Fourier transform, and Fourier convolution formula, the result is

$$
\begin{aligned}
& u(x, t)=F^{-1}[\tilde{u}(\lambda, t)] \\
& =F^{-1}\left[\frac{\sin \lambda a t}{\lambda a} \tilde{\psi}(\lambda)\right]+F^{-1}[\cos \lambda a t \tilde{\varphi}(\lambda)] \\
& =F^{-1}\left[\frac{\sin \lambda a t}{\lambda a}\right]^{*} \tilde{\psi}(x)+F^{-1}[\cos \lambda a t]^{*} \tilde{\varphi}(x) .
\end{aligned}
$$

vii. with MATLAB solve $F^{-1}\left[\frac{\sin \lambda a t}{\lambda a}\right], F^{-1}[\cos \lambda a t]$.

Input in the MATLAB window(with A,B instead of $F^{-1}\left[\frac{\sin \lambda a t}{\lambda a}\right], F^{-1}[\cos \lambda a t]$ ).

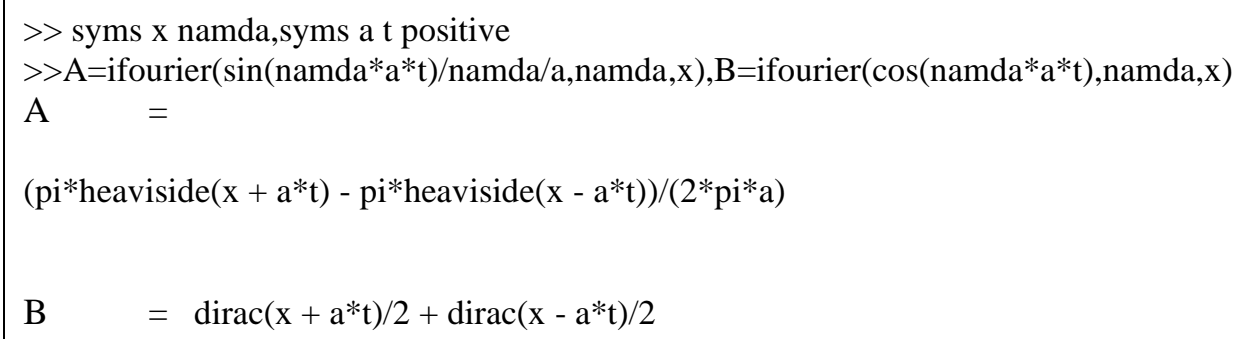

By using the unit step function, get

$$
\begin{gathered}
F^{-1}\left[\frac{\sin \lambda a t}{\lambda a}\right]=\frac{1}{2 a}[H(x+a t)-H(x-a t)] \\
F^{-1}[\cos \lambda a t]=\frac{1}{2}[\delta(x+a t)+\delta(x-a t)] .
\end{gathered}
$$

To bring it into the expression of $u(x, t)$ get

$$
u(x, t)=\frac{1}{2 a}[H(x+a t)-H(x-a t)] * \psi(x)+\frac{1}{2}[\delta(x+a t)+\delta(x-a t)] * \varphi(x) .
$$

For the above equation, Using the definition and properties of convolution, the definition of unit step function and the definition of a function 


$$
u(x, t)=\frac{1}{2}[\varphi(x+a t)+\varphi(x-a t)]+\frac{1}{2 a} \int_{x-a t}^{x+a t} \psi(\zeta) d \zeta .
$$

This is the solution to the problem of the definite solution of the free vibration of an unbounded string. This formula is the D'Alembert's formula.

\section{Summary}

Since the algorithm 2 is using MATLAB software, making computing easier than Algorithm 1.

\section{References}

[1].Yanping Bai. Equations of Mathematical Physics [M]. Beijing Institute of Technology Press, 2006.

[2].Xiayun Li. Algorithm infinite string free vibration problems [J]. Mathematical Theory and Applications, 2013(1):100103.

[3].Bracewell R. The Fourier transform and its applications [M].

[4].Brout R, Massar S, Parentani R, et al. Hawking radiation without trans-Planckian frequencies. [J]. Physical Review D Particles \& Fields, 1995, 52(8):4559-4568.

[5].Penney R C, Rukhin A L. D'Alembert's Functional Equation on Groups[J]. Proc.amer.math.soc, 2013, 99(99):73-80.

[6].Stilbs P. Fourier transform pulsed-gradient spin-echo studies of molecular diffusion[J]. Progress in Nuclear Magnetic Resonance Spectroscopy, 1987, 19(1):1-45. 